\title{
BIG BIASES AMONGST PRODUCTS OF TWO PRIMES
}

\author{
DAVID DUMMIT, ANDREW GRANVILLE, AND HERSHY KISILEVSKY
}

\begin{abstract}
We show that substantially more than a quarter of the odd integers of the form $p q$ up to $x$, with $p, q$ both prime, satisfy $p \equiv q \equiv 3(\bmod 4)$.
\end{abstract}

\section{INTRODUCTION}

There are roughly equal quantities of odd integers $n$ that are the product of two primes, $p$ and $q$, in the two arithmetic progressions $1(\bmod 4)$ and $3(\bmod 4)$. Indeed the counts differ by no more than $x^{1 / 2+o(1)}$ (assuming the Riemann Hypothesis for $L(1,(-4 /$.$) ); see$ [1] for a detailed analysis). One might guess that these integers are further evenly split amongst those with $(p(\bmod 4), q(\bmod 4))=(1,1),(1,-1),(-1,1)$ or $(-1,-1)$, but recent calculations reveal a substantial bias towards those $p q \leq x$ with $p \equiv q \equiv 3(\bmod 4)$. Indeed for the ratio

$$
r(x):=\#\{p q \leq x: p \equiv q \equiv 3(\bmod 4)\} / \frac{1}{4} \#\{p q \leq x\}
$$

we found that

$$
r(1000) \approx 1.347, r\left(10^{4}\right) \approx 1.258, r\left(10^{5}\right) \approx 1.212, r\left(10^{6}\right) \approx 1.183, r\left(10^{7}\right) \approx 1.162,
$$

a pronounced bias that seems to be converging to 1 surprisingly slowly. We will show that this is no accident and that there is similarly slow convergence for many such questions:

Theorem 1.1. Let $\chi$ be a quadratic character of conductor $d$. For $\eta=-1$ or 1 we have

$$
\frac{\#\{p q \leq x: \chi(p)=\chi(q)=\eta\}}{\frac{1}{4} \#\{p q \leq x:(p q, d)=1\}}=1+\eta \frac{\left(\mathcal{L}_{\chi}+o(1)\right)}{\log \log x} \text { where } \mathcal{L}_{\chi}:=\sum_{p} \frac{\chi(p)}{p} .
$$

If $\chi=(-4 /$.$) then \mathcal{L}_{\chi}=-.334 \ldots$ so the theorem implies that $r(x) \geq 1+\frac{(1+o(1))}{3(\log \log x-1)}$. If we let $s(x)=1+\frac{1}{3(\log \log x-1)}$ then we have

$$
s(1000) \approx 1.357, s\left(10^{4}\right) \approx 1.273, s\left(10^{5}\right) \approx 1.230, s\left(10^{6}\right) \approx 1.205, s\left(10^{7}\right) \approx 1.187,
$$

a pretty good fit with the data above. The prime numbers have only been computed up to something like $10^{24}$ so it is barely feasible that one could collect data on this problem up to $10^{50}$ in the foreseeable future. Therefore we would expect this bias to be at least $7 \%$ on any data that will be collected this century (as $\left.s\left(10^{50}\right) \approx 1.07\right)$.

Date: August 24, 2018.

2010 Mathematics Subject Classification. 11L20, 11N25, 11N13.

Key words and phrases. Primes in arithmetic progressions, Prime races. 
Proof. For a given quadratic Dirichlet character $\chi$ we will count the number of integers $p q \leq x$ with $\chi(p)=\chi(q)=1$ (and the analogous argument works for -1 ). One can write any such integer $p q \leq x$ with $p \leq q \leq x / p$, so that $p \leq \sqrt{x}$. Hence we wish to determine

$$
\sum_{\substack{p \leq \sqrt{x} \\ \chi(p)=1}} \sum_{\substack{p \leq q \leq x / p \\ \chi(q)=1}} 1
$$

The prime number theorem for arithmetic progressions reveals that $\sum_{q \leq Q, \chi(q)=1} 1=\frac{Q}{2 \log Q}+$ $O\left(\frac{Q}{(\log Q)^{2}}\right)$, so the above sum equals

$$
\sum_{p \leq \sqrt{x}} \frac{\left(\chi_{0}(p)+\chi(p)\right)}{2} \cdot \frac{x}{2 p \log (x / p)}+O\left(\frac{x}{p(\log x)^{2}}+\frac{p}{\log p}\right)
$$

where the implicit constant in the $O($.$) depends only on the conductor of \chi$. This equals

$$
\frac{1}{4} \sum_{\substack{p \leq \sqrt{x} \\(p, d)=1}} \frac{x}{p \log (x / p)}+\frac{x}{4} \sum_{p \leq \sqrt{x}} \frac{\chi(p)}{p \log (x / p)}+O\left(\frac{x}{(\log x)^{2}} \log \log x\right) .
$$

The difference between the second sum, and the same sum with $\log (x / p)$ replaced by $\log x$, is

$$
\frac{x}{4 \log x} \sum_{p \leq \sqrt{x}} \frac{\chi(p)}{p \log (x / p)} \ll \frac{x}{(\log x)^{2}} .
$$

using the prime number theorem for arithmetic progression and partial summation. Moreover

$$
\frac{x}{4 \log x} \sum_{p>\sqrt{x}} \frac{\chi(p)}{p} \ll \frac{x}{(\log x)^{2}} .
$$

Collecting together what we have proved so far yields that $\#\{p q \leq x: \chi(p)=\chi(q)=1\}$

$$
=\frac{1}{4}\left\{\#\{p q \leq x:(p, d)=1\}+\frac{x}{\log x} \sum_{p} \frac{\chi(p)}{p}+O\left(\frac{x}{(\log x)^{2}} \log \log x\right)\right\}
$$

The first term is well-known to equal $\frac{x}{\log x}(\log \log x+O(1))$, and so we deduce that

$$
\frac{4 \#\{p q \leq x: \chi(p)=\chi(q)=1\}}{\#\{p q \leq x:(p q, d)=1\}}=1+\frac{1}{\log \log x}\left(\sum_{p} \frac{\chi(p)}{p}+o(1)\right) .
$$

as claimed.

We note that

$$
\sum_{p} \frac{\chi(p)}{p}=\sum_{m \geq 1} \frac{\mu(m)}{m} \log L\left(m, \chi^{m}\right)=\log L(1, \chi)+E(\chi),
$$

where

$$
\sum_{p}\left(\log \left(1-\frac{1}{p}\right)+\frac{1}{p}\right)=-0.315718 \ldots \leq E(\chi) \leq \sum_{p}\left(\log \left(1+\frac{1}{p}\right)-\frac{1}{p}\right)=-0.18198 \ldots
$$




\section{FURTHER REMARKS}

- One deduces from our theorem that $r(x)>1$ for all $x$ sufficiently large and we conjecture that this is true for all $x \geq 9$.

- We also conjecture that $\mathcal{L}_{\chi}$ is always non-zero so that there is always such a bias.

- One can calculate the bias in other such questions. For example, we get roughly triple the bias for the proportion of $p q \leq x$ for which $\left(\frac{p}{5}\right)=\left(\frac{q}{5}\right)=-1$ out of all $p q \leq x$ with $p, q \neq 5$ (since $\left.\mathcal{L}_{(. / 5)} \approx-1.008\right)$. The data

$$
r_{5}(1000) \approx 1.881, r_{5}\left(10^{4}\right) \approx 1.626, r_{5}\left(10^{5}\right) \approx 1.523, r_{5}\left(10^{6}\right) \approx 1.457, r_{5}\left(10^{7}\right) \approx 1.416,
$$

confirms this very substantial bias. It would be interesting to find more extreme examples.

- How large can the bias get if $d \leq x$ ? It is known [2] that $L(1, \chi)$ can be as large as $c \log \log d$, and so $\mathcal{L}_{\chi}$ can be as large as $\log \log \log d+O(1)$. We conjecture that there exists $d \leq x$ for which the bias in our Theorem is as large as

$$
1+\frac{\log \log \log x+O(1)}{\log \log x} .
$$

Note that this requires proving a uniform version of the Theorem. Our proof assumes that $x$ is allowed to be very large compared to $d$, so does not immediately apply to the problem that we have just stated.

- The same bias can be seen (for much the same reason) in looking at

$$
\sum_{\substack{p \leq x \\ p \equiv 3(\bmod 4)}} \frac{1}{p} / \sum_{\substack{p \leq x \\ p \equiv 1(\bmod 4)}} \frac{1}{p} \approx 1+\frac{2}{3 \log \log x} .
$$

Indeed, by the analogous proof, we have in general

$$
\sum_{\substack{p \leq x \\ \chi(p)=1}} \frac{1}{p} / \sum_{\substack{p \leq x \\ \chi(p)=-1}} \frac{1}{p}=1+2 \frac{\left(\mathcal{L}_{\chi}+o(1)\right)}{\log \log x} .
$$

We therefore see a bias in the distribution of primes in arithmetic progressions, where each prime $p$ is weighted by $1 / p$, as a consequence of the sign of $\mathcal{L}_{\chi}$. This effect is much more pronounced than in the traditional prime race problem where the same comparison is made, though with each prime weighted by 1 . The bias here is determined by the distribution of values of $\chi(p)$, whereas the prime race bias is determined by the values of $\chi\left(p^{2}\right)=1$, so they appear to be independent phenomena. However one might guess that both biases are sensitive to low lying zeros of $L(s, \chi)$. This probably deserves further investigation, to determine whether there are any correlations between the two biases.

- One can show the following for $k$ prime factors, by similar methods:

$$
\frac{\#\left\{p_{1} \ldots p_{k} \leq x: \text { each } \chi\left(p_{j}\right)=\eta\right\}}{\frac{1}{2^{k}} \#\left\{p_{1} \ldots p_{k} \leq x: \operatorname{each}\left(p_{j}, d\right)=1\right\}}=1+\eta \frac{\left((k-1) \mathcal{L}_{\chi}+o(1)\right)}{\log \log x}
$$

It would be interesting to understand this when $k$ gets large, particularly when $k \sim \log \log x$, the typical number of prime factors of an integer $\leq x$. It seems likely that the factor on the 
right-side should grow like

$$
c_{\chi, k}\left(1+\eta \frac{\left(\mathcal{L}_{\chi}+o(1)\right)}{\log \log x}\right)^{k-1}
$$

but we do not know what $c_{\chi, k}$ would look like.

- More generally, if $\chi_{1}, \ldots, \chi_{k}$ are quadratic characters (with $\chi_{j}$ of conductor $d_{j}$ ), and each $\eta_{j}=-1$ or 1 then

$$
\frac{\#\left\{p_{1} \ldots p_{k} \leq x: \chi_{j}\left(p_{j}\right)=\eta_{j} \text { for each } j\right\}}{\frac{1}{2^{k}} \#\left\{p_{1} \ldots p_{k} \leq x: \text { each }\left(p_{j}, d_{j}\right)=1\right\}}=1+\frac{((k-1) c(\vec{\chi}, \vec{\eta})+o(1))}{\log \log x}
$$

where

$$
c(\vec{\chi}, \vec{\eta}):=\frac{1}{k} \sum_{j=1}^{k} \eta_{j} \mathcal{L}_{\chi_{j}}
$$

In particular this type of bias does not appear when $k=2, \chi_{1}=\chi_{2}$ and $\eta_{1}+\eta_{2}=0$. Can one prove that $c(\vec{\chi}, \vec{\eta})$ can only be 0 for such trivial reasons? That is, is $c(\vec{\chi}, \vec{\eta})=0$ if and only if $\sum_{j: \chi_{j}=\chi} \eta_{j}=0$ for every character $\chi \in \vec{\chi}$ ?

- Given arithmetic progressions $a(\bmod m)$ and $b(\bmod n)$, one can surely prove that there exists $\beta=\beta(a(\bmod m), b(\bmod n))$ such that

$$
\frac{\#\{p q \leq x: p \equiv a(\bmod m), q \equiv b(\bmod n)\}}{\frac{1}{\phi(m) \phi(n)} \#\{p q \leq x:(p, m)=(q, n)=1\}}=1+\frac{\beta+o(1)}{\log \log x} .
$$

It would be interesting to classify when $\beta(a(\bmod m), b(\bmod n))$ is non-zero, and to determine situations in which it is large. Or more generally for what subsets $A \subseteq(\mathbb{Z} / m \mathbb{Z})^{*}$ and $B \subseteq(\mathbb{Z} / n \mathbb{Z})^{*}$ is there no such bias? We would guess that this would only be the case if either

(i) $A$ and $B$ both contain all congruence classes (that is, every prime not dividing $m n$ can be represented by both $A$ and $B$ ); or

(ii) $A \cup B$ is a partition of the integers coprime to $m n$ (that is, every prime not dividing $m n$ is represented by $A$, or represented by $B$, but not both).

\section{REFERENCES}

[1] Kevin Ford and Jason Sneed, Chebyshev's bias for products of two primes, Experiment. Math. 19 (2010), 385-398.

[2] Andrew Granville and K. Soundararajan, The distribution of values of $L\left(1, \chi_{d}\right)$, Geom. Funct. Anal. 13 (2003), 992-1028.

Department of Mathematics and Statistics, University of Vermont, Burlington, Vt 05401, USA

E-mail address: dummit@math.uvm.edu

Centre de recherches mathématiques, Université de Montréal, CP 6128 succ. CentreVille, Montréal, QC H3C 3J7, Canada

E-mail address: andrew@dms.umontreal.ca

Department of Mathematics and Statistics, Sir George Williams Campus, Concordia University, Montreal, QC H3G 1M8, Canada

E-mail address: kisilev@mathstat.concordia.ca 